\title{
Overexpression of GMNFYA5 confers drought tolerance to transgenic Arabidopsis and soybean plants
}

\section{CURRENT STATUS: ACCEPTED}

BMC Plant Biology $\mathbf{B} M C$ Series

Xiao-Jun Ma

Northeast Agricultural University

Tai-Fei Yu

Institute of Crop Sciences, Chinese Academy of Agricultural Sciences

Xiao-Hui Li

Jilin Academy of Agricultural Sciences

Xin-You Cao

Shandong Academy of Agricultural Sciences

Jian Ma

Jilin Agricultural University

Jun Chen

Institute of Crop Science, Chinese Academy of Agricultural Sciences

Yong-Bin Zhou

Institute of crop science, Chinese Academy of Agricultural Sciences

Ming Chen

Institute of Crop Science, Chinese Academy of Agricultulral Sciences

You-Zhi Ma

Institute of Crop Science, Chinese Academy of Agricultural sciences

Jun-Hua Zhang

Northeast Agricultural University

Zhao-Shi Xu

Institute of Crop Science, Chinese Academy of Agricultural Sciences

xuzhaoshi@caas.cnCorresponding Author

ORCiD: https://orcid.org/0000-0001-8028-6413 


\section{DOI:}

$10.21203 / \mathrm{rs} .2 .17613 / \mathrm{v} 1$

\section{SUBJECT AREAS}

Plant Molecular Biology and Genetics

\section{KEYWORDS}

ABA sensitivity, Glycine max , Nuclear Factor YA, resistance mechanisms 
Abstract

Background: Crop productivity is challenged by abiotic stresses, among which drought stress is the most common. NF-Y genes, especially NF-YA genes, regulate tolerance to abiotic stress.

Results: Soybean NF-Y gene GmNFYA5 was identified to have the highest transcript level among all 21 NF-YA genes in soybean ( Glycine max L.) under drought stress. Drought-induced transcript of GmNFYA5 was suppressed by the ABA synthesis inhibitor naproxen (NAP). GmNFYA5 transcript was detected in various tissues at vegetative and reproductive growth stages with higher levels in roots and leaves than in other tissues, which was consist with the GmNFYA5 promoter:GUS fusion assay. Overexpression of GmNFYA5 in transgenic Arabidopsis plants caused enhanced drought tolerance in seedlings by decreasing stomatal aperture and water loss from leaves. Overexpression and suppression of GmNFYA5 in soybean resulted in increased and decreased drought tolerance, respectively, relative to plants with an empty vector (EV). Transcript levels of ABA-dependent genes ( ABI2 , ABI3 , NCED3 , LEA3 , RD29A , P5CS1 , GmWRKY46 , GmNCED2 and GmbZIP1 ) and ABAindependent genes ( DREB1A , DREB2A, DREB2B , GmDREB1 , GmDREB2 and GmDREB3 ) in transgenic plants overexpressing GmNFYA5 were higher than those of wild-type plants under drought stress; suppression of GmNFYA5 transcript produced opposite results. GmNFYA5 probably regulated the transcript abundance of GmDREB2 and GmbZIP1 by binding to the promoters in vivo.

Conclusions: Our results suggested that overexpression of GmNFYA5 improved drought tolerance in soybean via both ABA-dependent and ABA-independent pathways.

\section{Full Text}

Due to technical limitations, full-text HTML conversion of this manuscript could not be completed. However, the manuscript can be downloaded and accessed as a PDF.

\section{Figures}
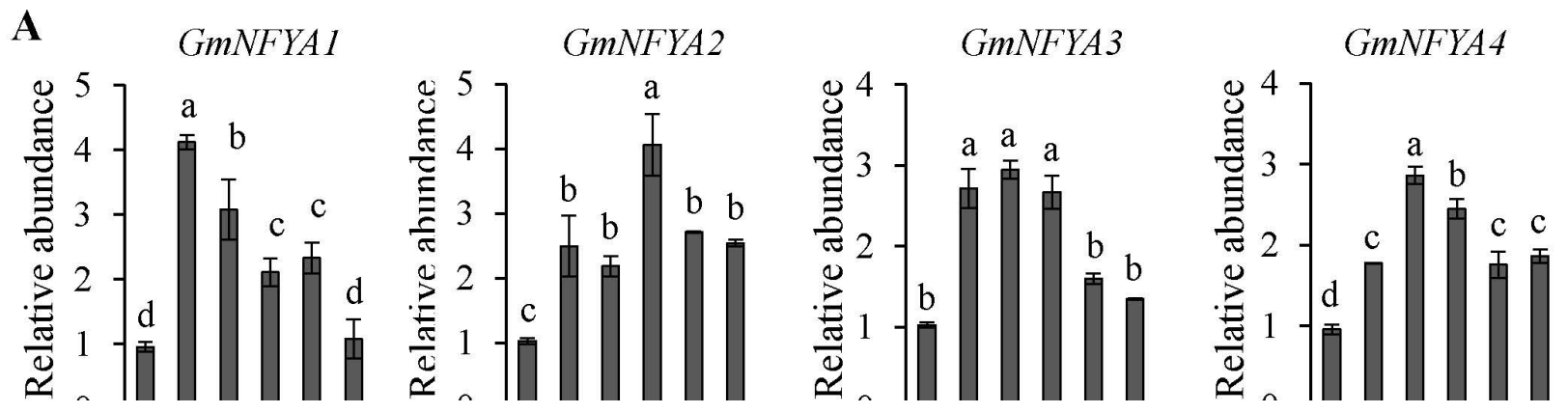


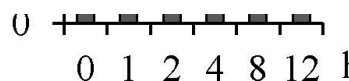

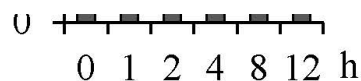

מש,

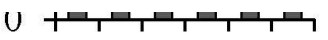

GmNFYA5

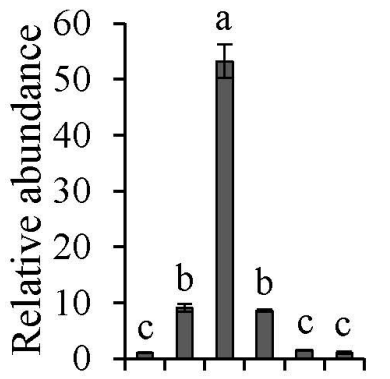

$0124812 \mathrm{~h}$
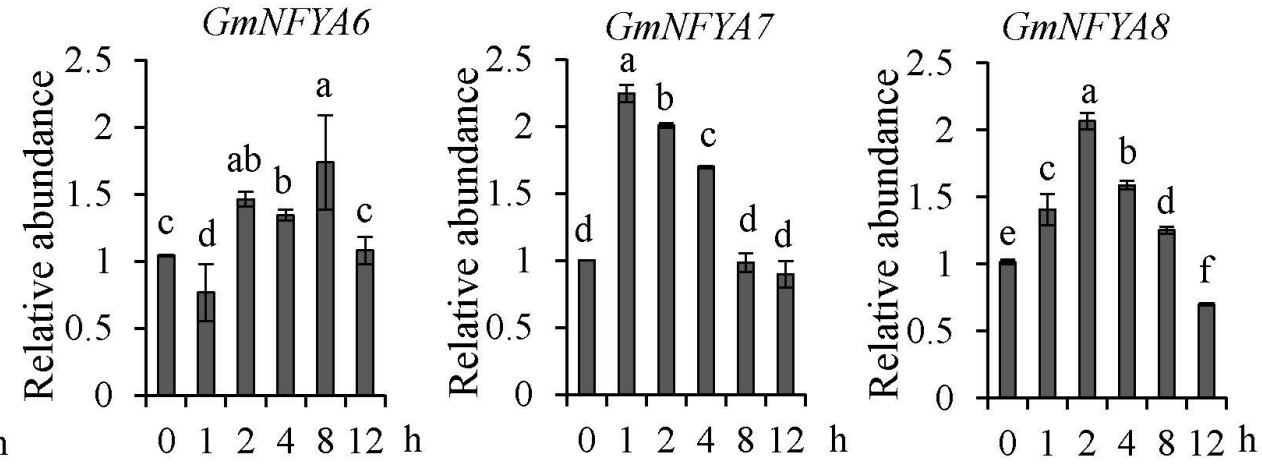

GmNFYA9
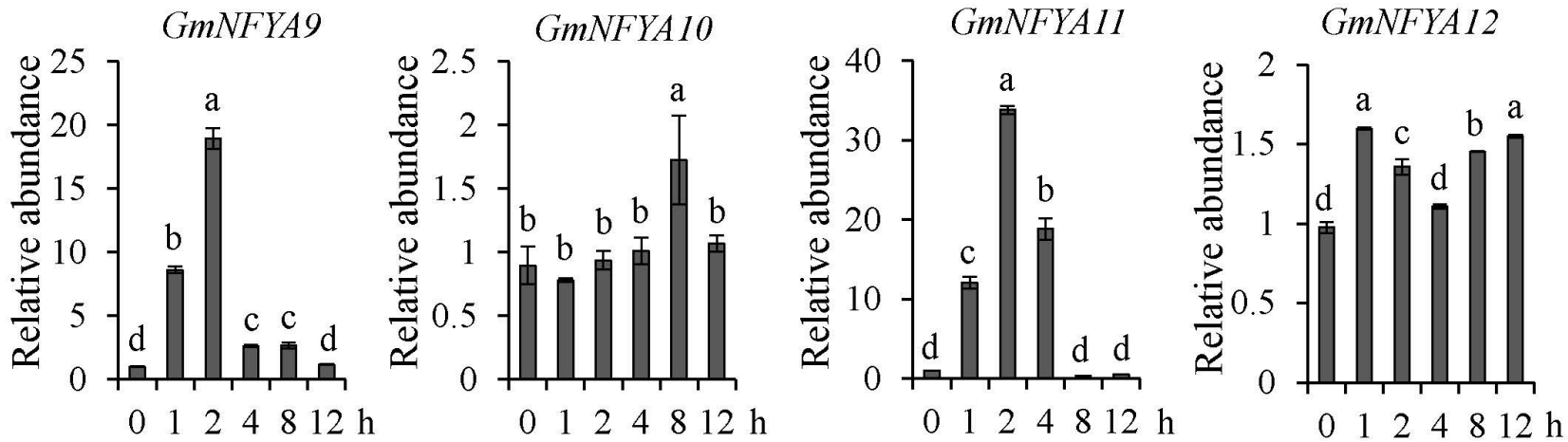

GmNFYA13

GMNFYA14
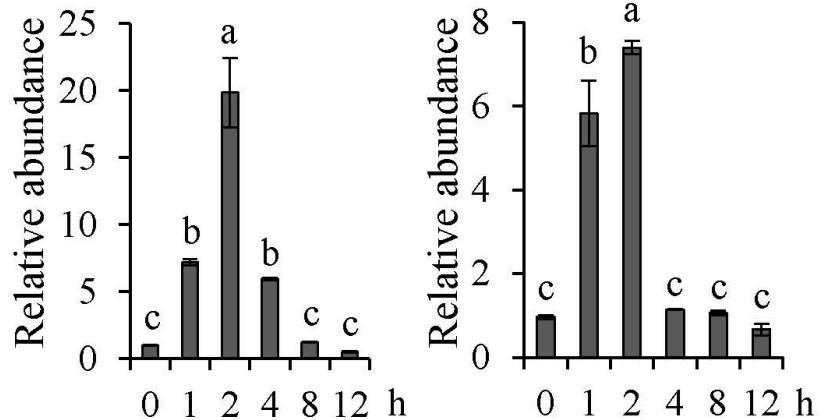

GmNFYA17

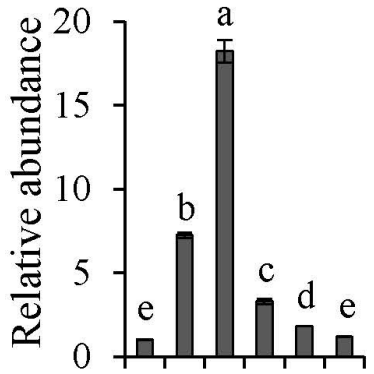

$0124812 \mathrm{~h}$

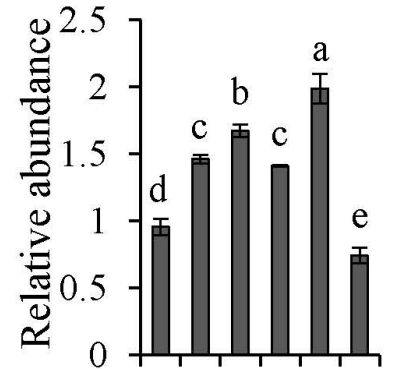

$0124812 \mathrm{~h}$
GmNFYA15

GmNFYA16
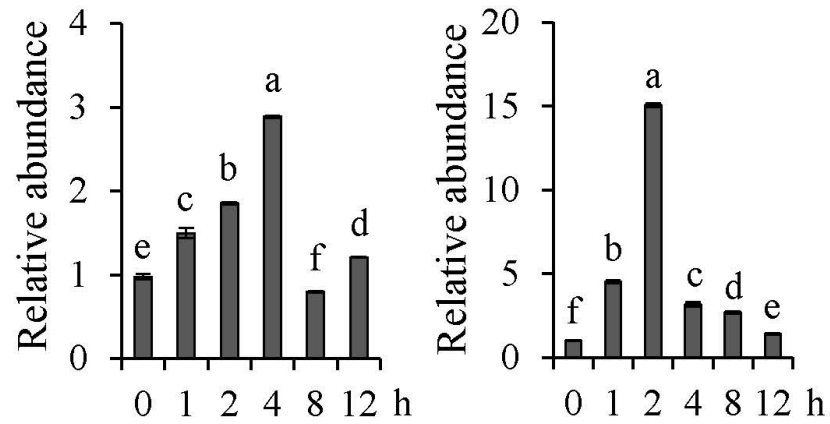

GmNFYA19

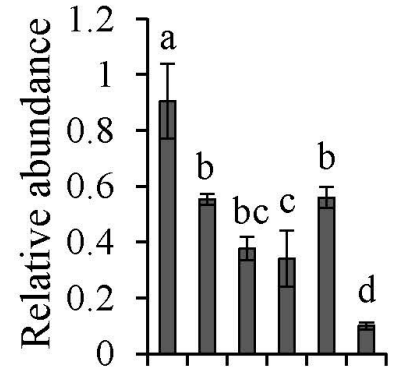

$0 \begin{array}{lllll}0 & 2 & 4 & 812 \mathrm{~h}\end{array}$

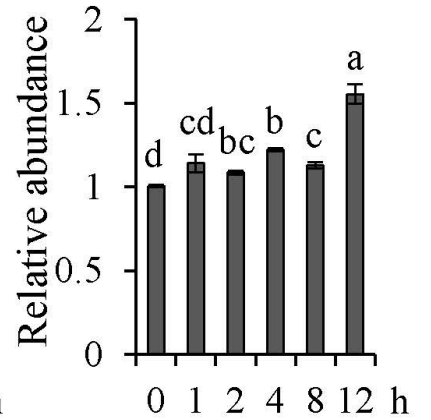

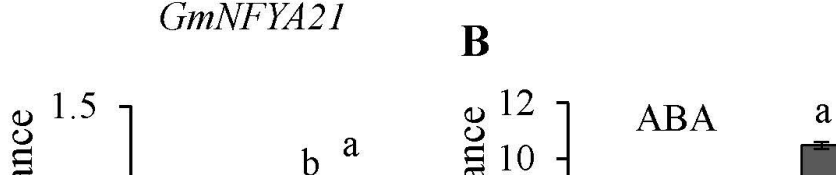

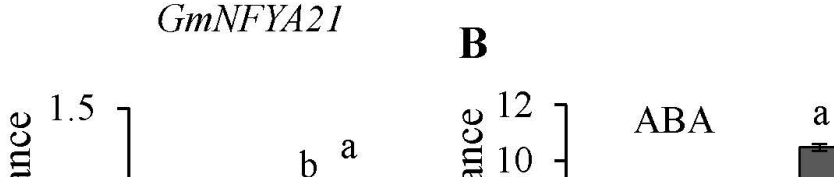

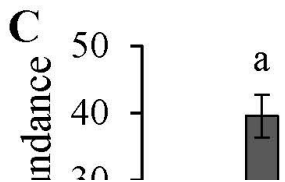



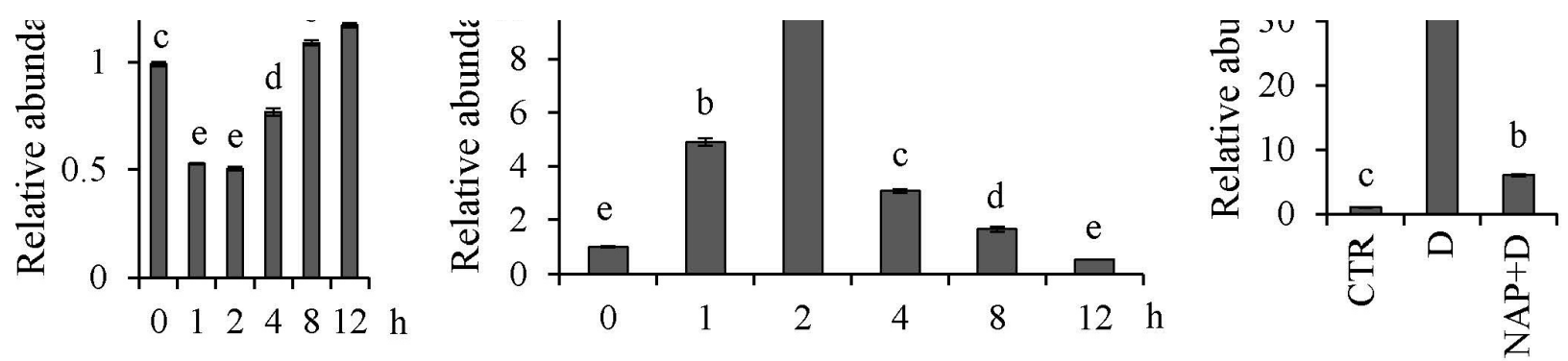

Figure 1

GmNFYA5 expression as affected by drought stress and ABA. (A) Transcripts of 21 NF-YA genes were analyzed using qRT-PCR in soybean under drought stress. Expression levels were normalized to that of GmCYP2. GmNFYA5 transcript at $0 \mathrm{~h}$ was set at 1.0, and SD for three biological replicates is represented by error bars. (B) Transcript of GmNFYA5 analyzed in response to $A B A$ treatment in soybean. Expression levels were normalized to that of GmCYP2. GmNFYA5 transcript at $0 \mathrm{~h}$ was set at 1.0, and SD for three biological replicates is represented by error bars. (C) qRT-PCR of GmNFYA5 transcript in soybean plants in response to drought stress with the treatment of $1 \mathrm{mM} \mathrm{NAP}$. Control, drought and drought + NAP are indicated by CTR, D and D + NAP, respectively. The level of GmNFYA5 transcript under control conditions was set at 1.0, and the internal control was GmCYP2. SD for three biological replicates is represented by error bars. Significant differences at $\mathrm{P}<0.05$ are indicated by different letters above the columns. 

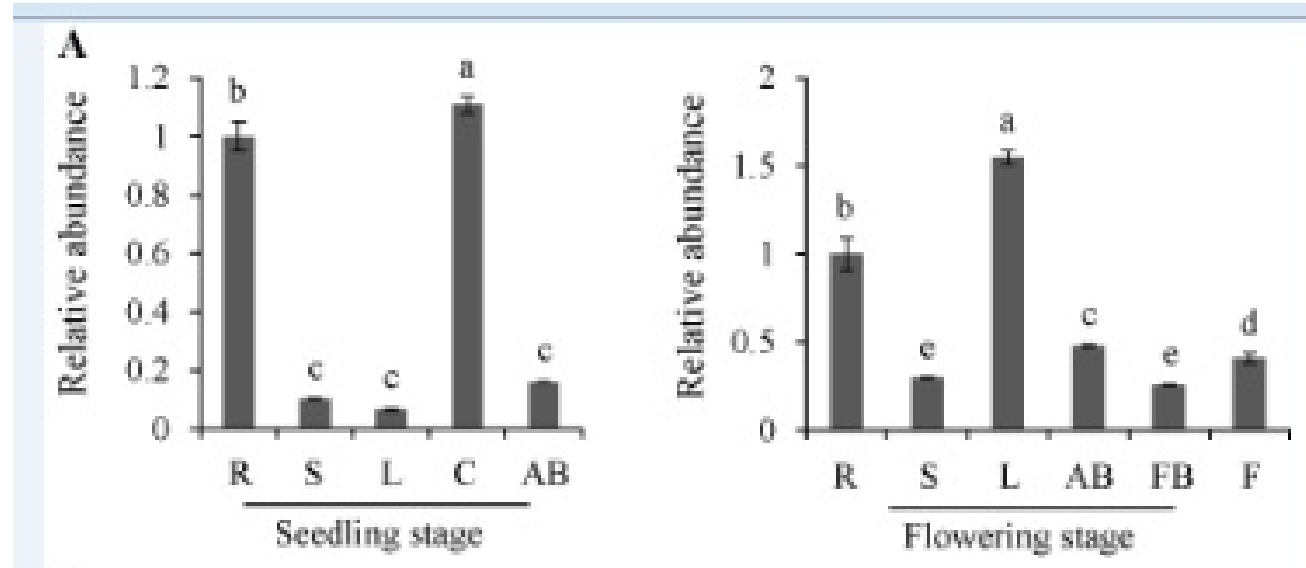

B
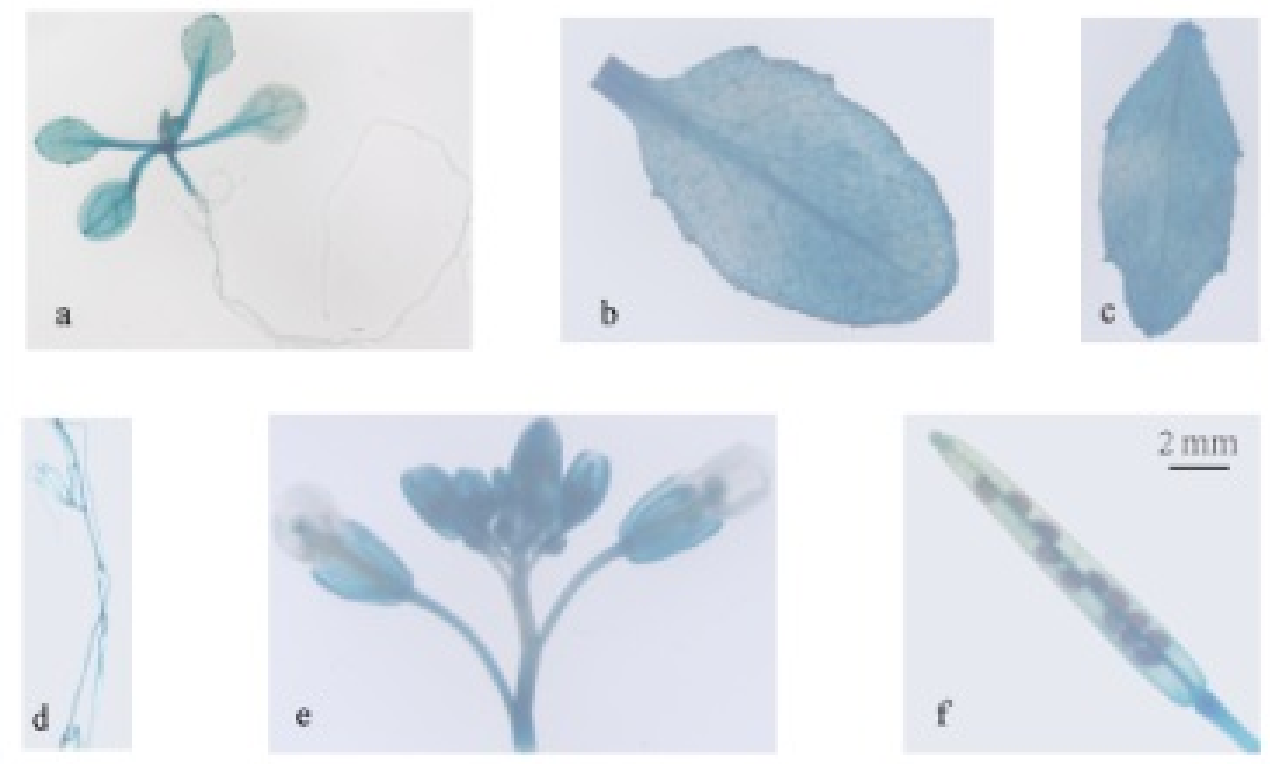

\section{GFP Bright field Chlorophyll $\quad$ Merged}
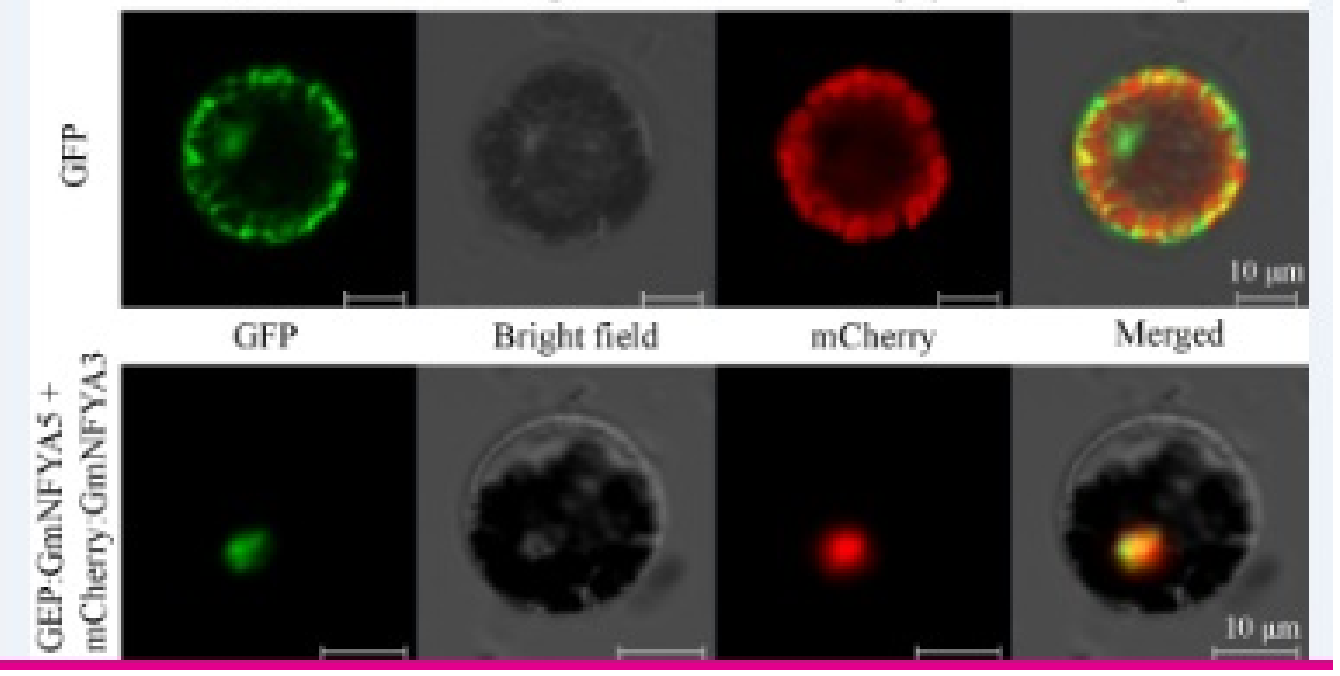

Figure 2

Tissue specific expression analysis and subcellular localization of GmNFYA5. (A) Expression 
abundance of the GmNFYA5 gene in soybean tissues at the seedling and flowering stages.

Soybean tissues under normal conditions were used to extract total RNA. The relative

transcript levels of GmNFYA5 in soybean tissues are indicated by vertical columns.

GmNFYA5 transcript in roots was set at 1.0, and the internal control was GmCYP2. SD for three biological replicates is represented by error bars. Significant differences at $\mathrm{P}<0.05$ are indicated by different letters above the columns. (B) Expression pattern of GmNFYA5 promoter:GUS in various tissues of transgenic Arabidopsis plants. a 5-day-old seedling, b rosette leaf, c cauline leaf, d root, e flower, f silique. Scale bar, $2 \mathrm{~mm}$ (C) Subcellular localization of GmNFYA5. Fluorescence of GFP-GmNFYA5 and mCherry-GmNFYA3 fusion proteins in transformed cells was localized exclusively to the nucleus. Scale bar, $10 \mu \mathrm{m}$.
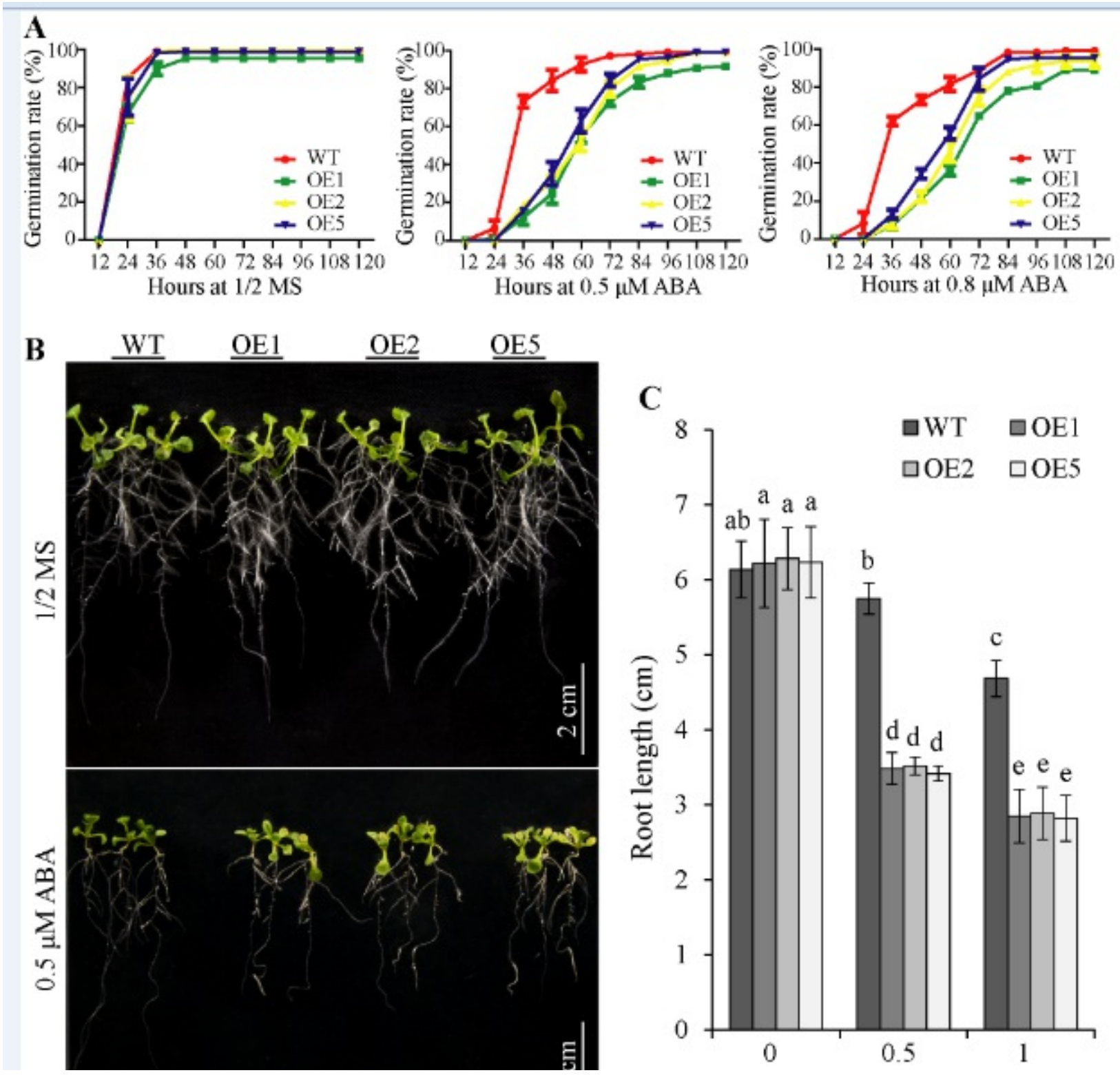
Figure 3

Germination and root growth of WT and transgenic Arabidopsis plants with ABA treatment. (A) Seed germination on 1/2 MS agar plates with 0, 0.5 and $0.8 \mu \mathrm{M}$ ABA. Each measurement represents the average germination of 64 seeds \pm SD. (B) and (C) Root growth of WT and transgenic Arabidopsis plants on media with/without ABA. Three-day-old seedlings from 1/2 MS medium were transferred to media containing $0,0.5$ and $1 \mu \mathrm{M} A B A$; and photographed 7 days later. Scale bar, $2 \mathrm{~cm}$. Each measurement represents the average root length of 30 seedlings \pm SD. Significant differences $P<0.05$ are indicated by different letters above the columns. 

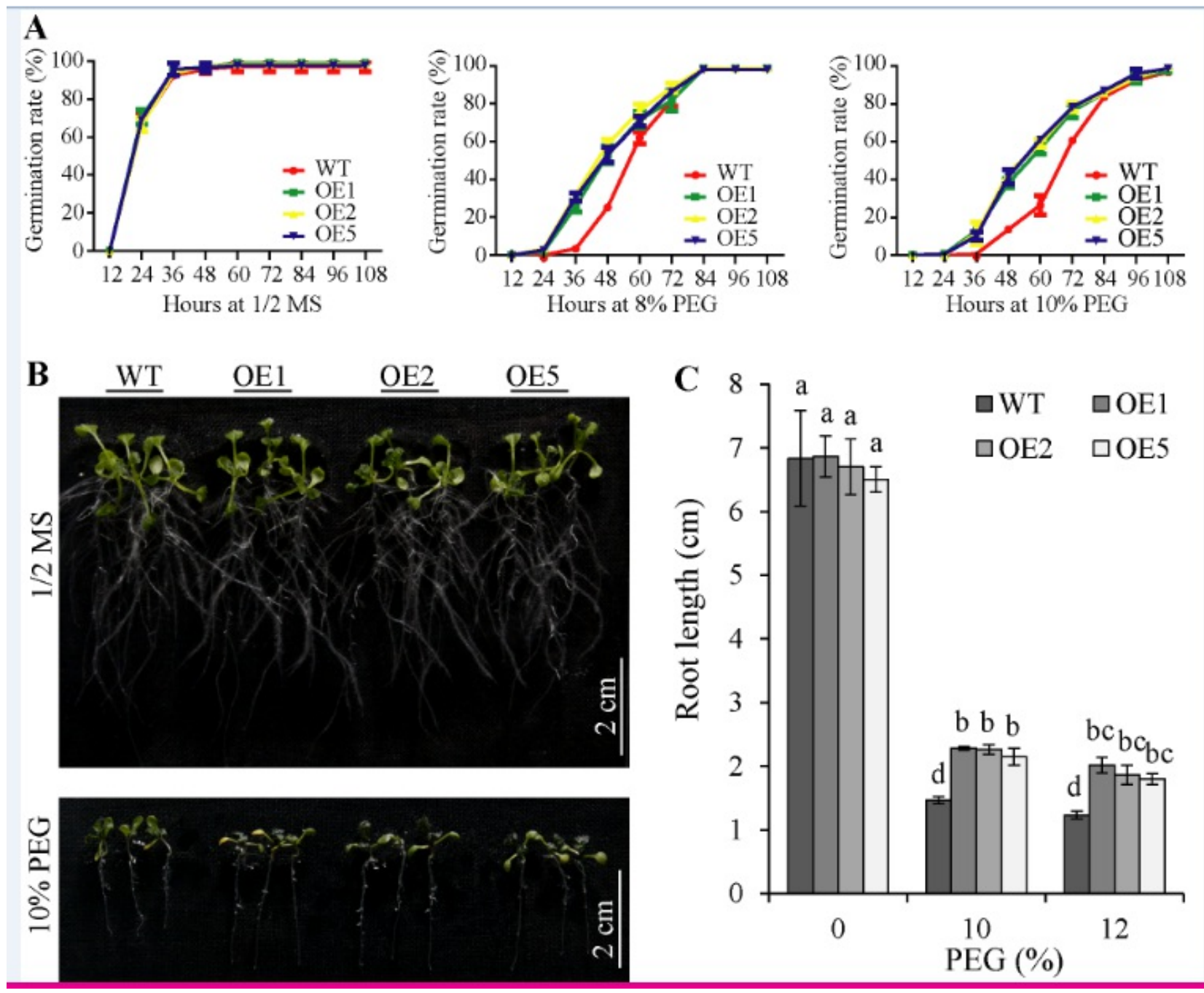

Figure 4

Germination rates and root growth of WT and transgenic Arabidopsis plants treated with PEG. (A) Seed germination on 1/2 MS agar plates with 8 and 10\% PEG. Each measurement represents the average germination of 64 seeds \pm SD. (B) and (C) Root growth of WT and transgenic Arabidopsis plants on media with/without PEG. Three-day-old seedlings from 1/2 MS medium were transferred to media containing 0,10 and 12\% PEG; photographed after 7 days. Scale bar, $2 \mathrm{~cm}$. Each measurement represents the average root length of 30 seedlings \pm SD. Significant differences at $P<0.05$ are indicated by different letters above the columns. 


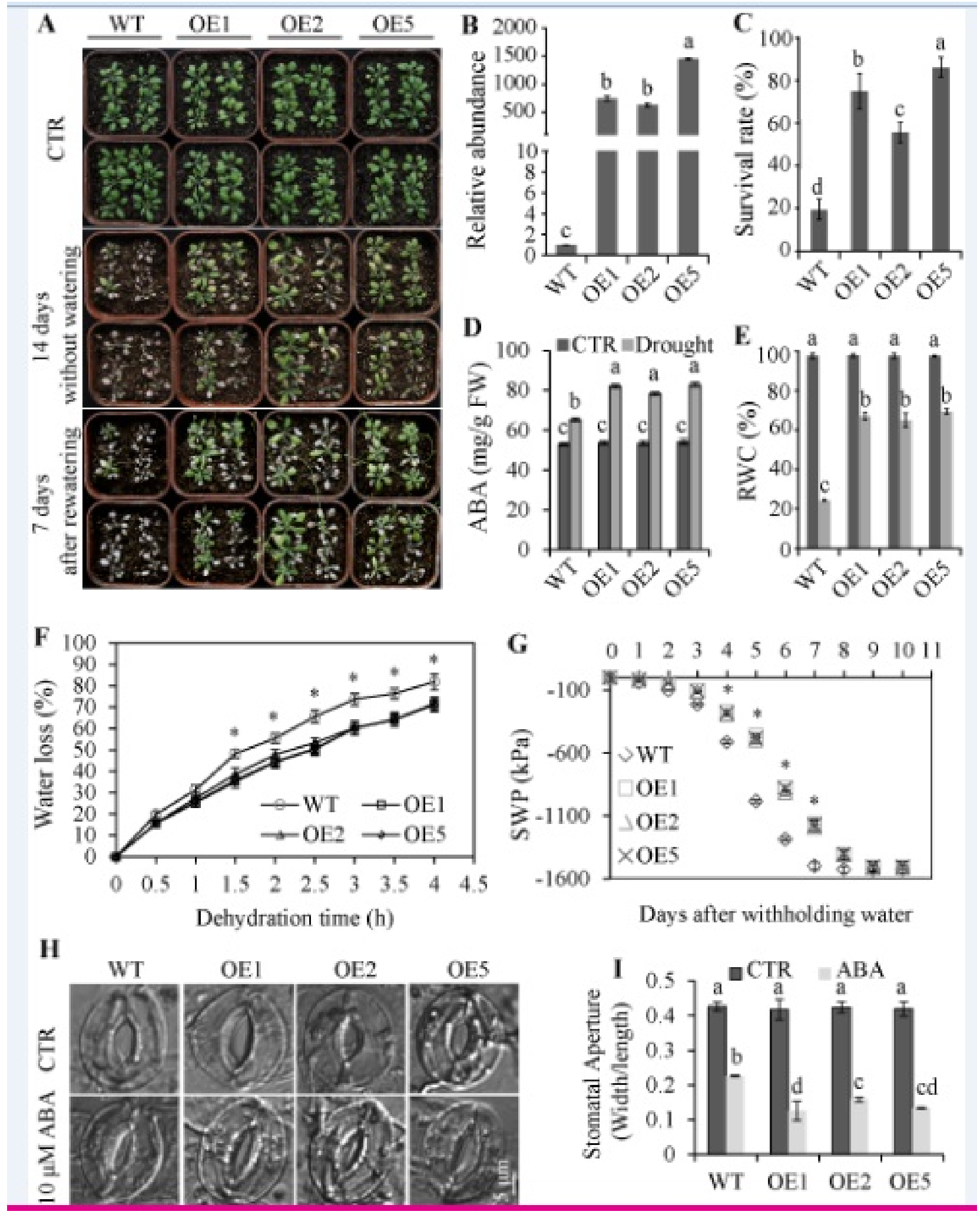

Figure 5

Improved drought tolerance and stomatal aperture analysis in transgenic Arabidopsis plants. (A) Assessment of drought tolerance in transgenic Arabidopsis plants. Three-weekold plants were grown without water for 14 days, followed by re-watering for 7 days. 
Drought resistance of transgenic Arabidopsis plants was assayed by capability to resume growth when returned to normal conditions after drought stress. (B) GmNFYA5 transcript detected in transgenic Arabidopsis lines. Expression levels were normalized to that of Tub8. Transcript of GmNFYA5 in WT plants was set at 1.0; SD for three biological replicates is represented by error bars. (C-G) Measurements of survival rate, concentrations of ABA, RWC, water loss, and SWP in transgenic Arabidopsis and WT lines. (H-I) Stomatal apertures in transgenic Arabidopsis and WT plants with zero and $10 \mu \mathrm{M}$ ABA treatments. Width/length of the stomatal aperture was measured using the ruler tool in Adobe Photoshop CS5. Scale bar, $5 \mu \mathrm{m}$. Data represent mean SD for three biological replicates ( $n=54$ ). Significant differences $\mathrm{P}<0.05$ are indicated by different letters above the columns. 

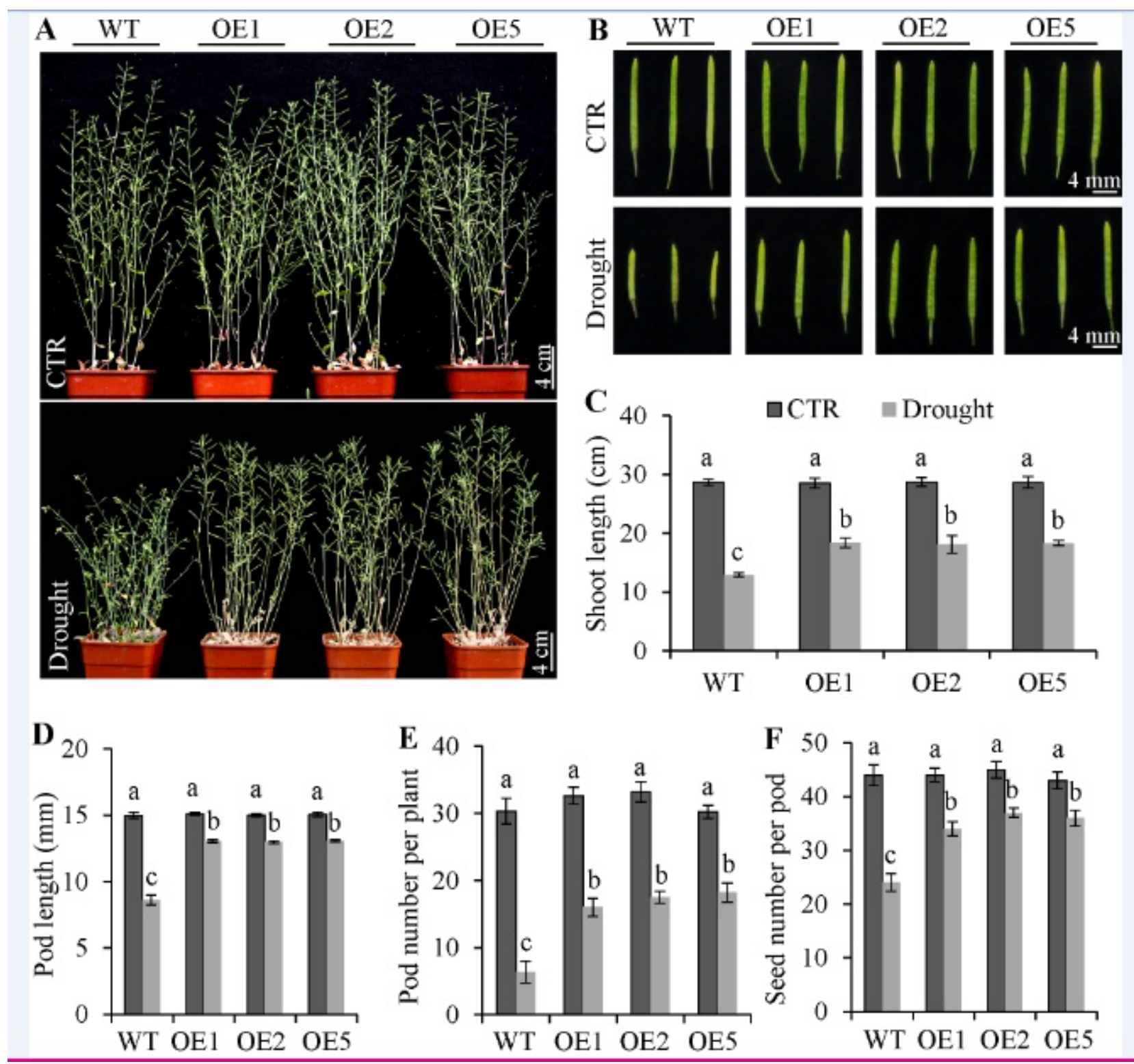

Figure 6

Improved drought tolerance in transgenic Arabidopsis plants all over an entire growth cycle.

(A) Drought tolerance assessed in transgenic Arabidopsis over a full growth cycle. Scale bar,

$4 \mathrm{~cm}$. (B) Pod lengths in transgenic and WT Arabidopsis plants were from control and drought conditions. Scale bar, $4 \mathrm{~mm}$. (C-F) Shoot length, pod length, pod number per plant and seed number per pod were measured in transgenic and WT plants under control and drought conditions. Data represent mean SD for three biological replicates $(n=18)$.

Significant differences at $\mathrm{P}<0.05$ are indicated by different letters above the columns.

\begin{tabular}{|c|c|c|c|c|c|}
\hline $\mathbf{A}$ & DREBIA & B & $D R E B 2 A$ & C & $D R E B 2 B$ \\
\hline$\left.\stackrel{\mathscr{E}}{ }^{20}\right\rceil \square \mathrm{OE} 1$ & $\begin{array}{l}\mathrm{a} \\
\mathrm{I}\end{array}$ & 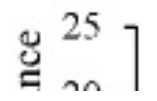 & $\begin{array}{l}\mathrm{a} \\
\mathrm{I}\end{array}$ & 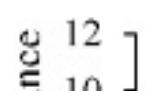 & $\mathrm{a}$ \\
\hline
\end{tabular}



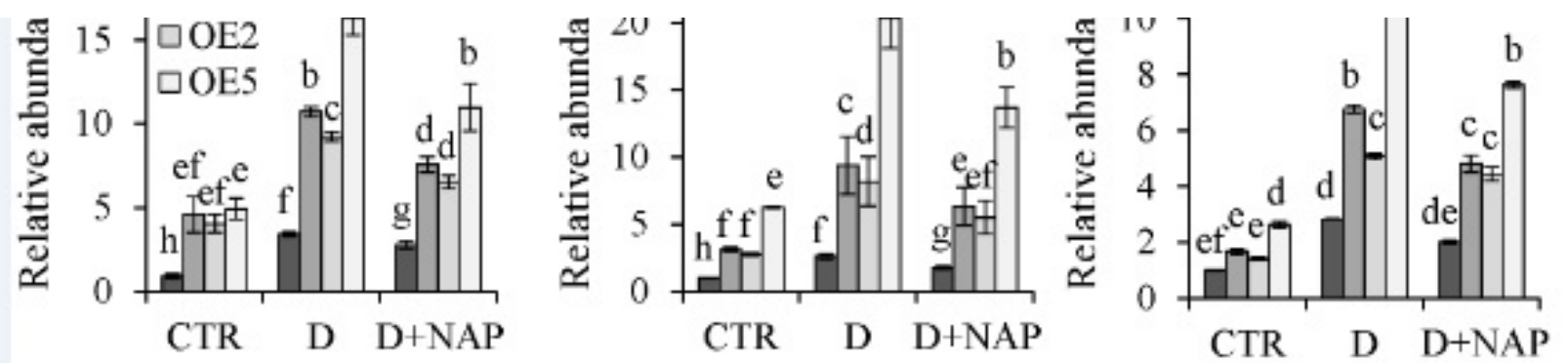

D

NCED 3

E

ABI2

F

ABI3
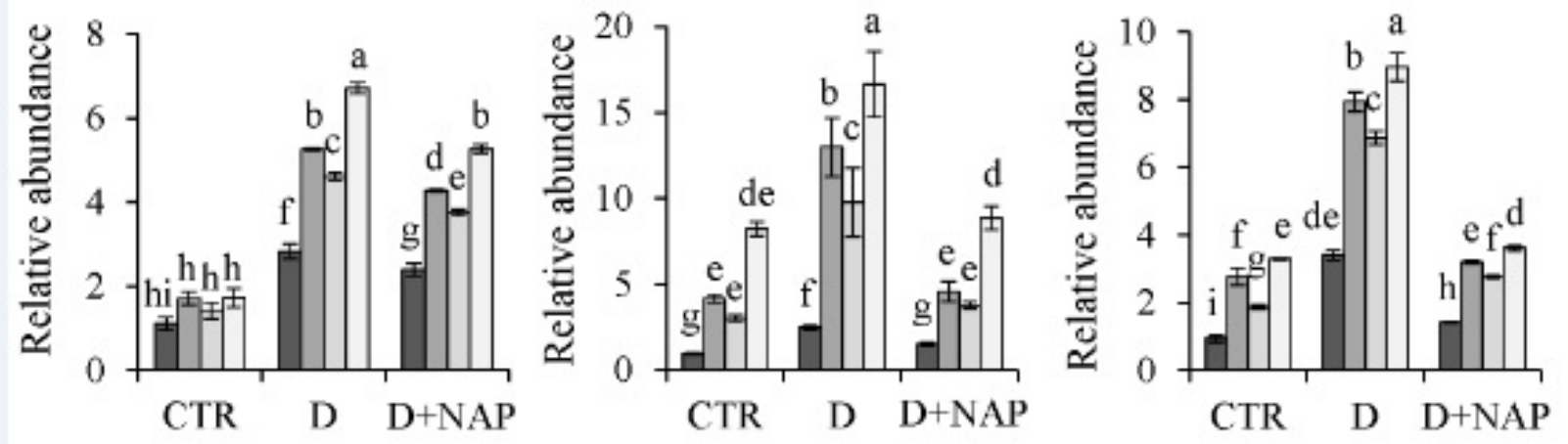

G

LEA3

H

RD29A

P5CSI
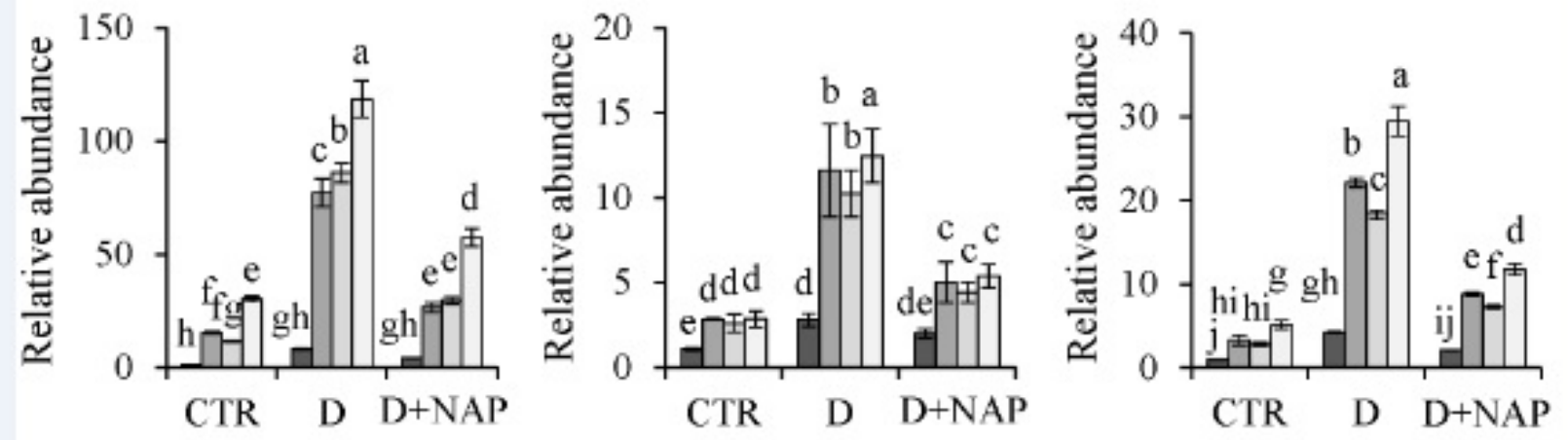

Figure 7

Relative transcript levels of DREB1A, DREB2A, DREB2B, ABI2, ABI3, NCED3, LEA3, RD29A

and P5CS1 in transgenic Arabidopsis and WT plants under three conditions. Relative

transcript level is indicated by the vertical column and normalized to that of Tub8.

Transcripts of stress-related genes in WT plants under normal conditions were set at 1.0.

Control, drought and drought pretreated with NAP are indicated by CTR, D and D + NAP, respectively. Data represent mean SD for three biological replicates. Significant differences $P<0.05$ are indicated by different letters above the columns. 


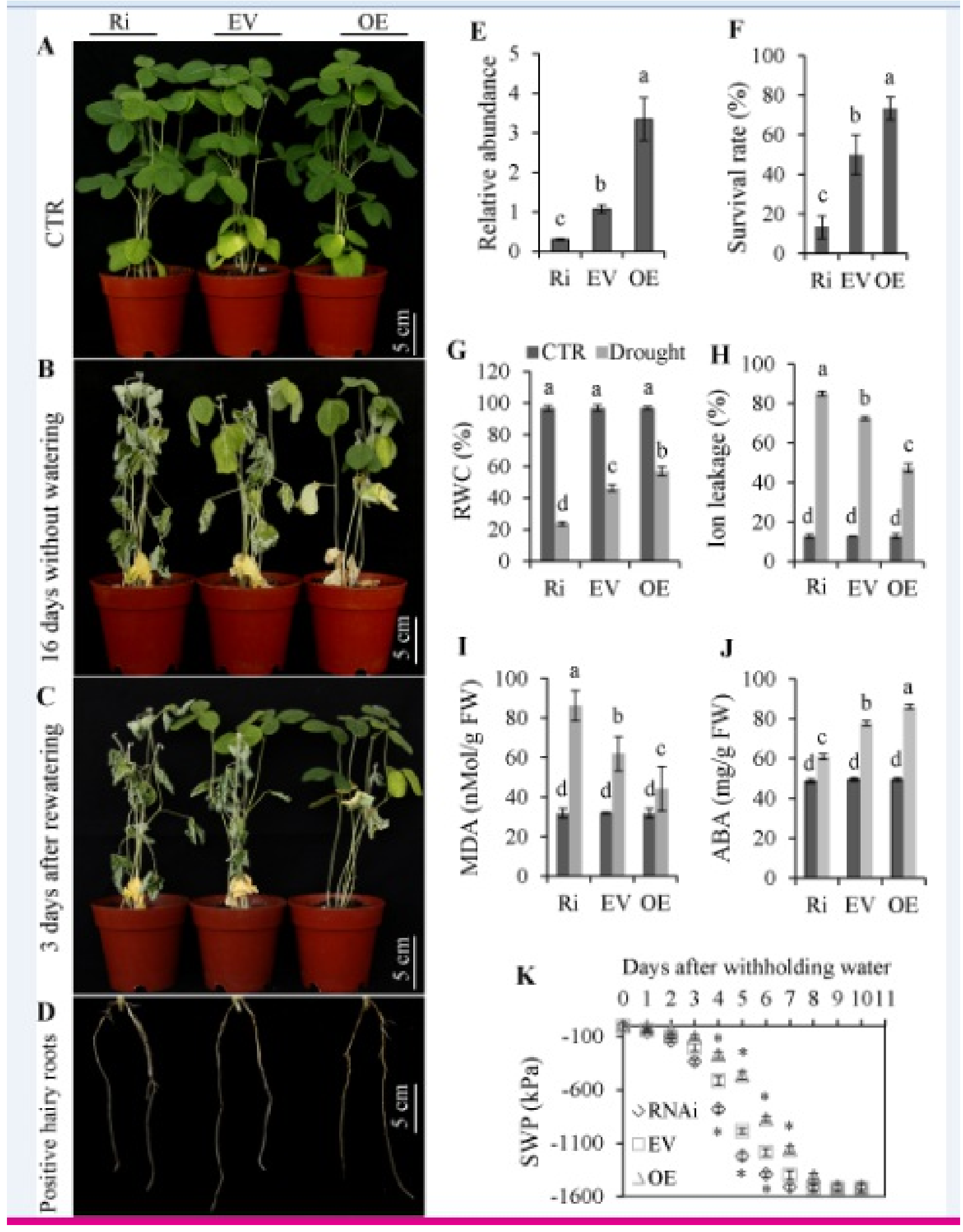

Figure 8

Drought tolerance in transgenic soybean plants. (A-D) Drought tolerance was evaluated in transgenic soybean plants. Transgenic soybean plants with positive hairy roots were 
transferred to plastic pots containing a mixture of peat and vermiculite $(1: 1, v / v)$ and grown for 7 days. Water deprivation for 16 days was followed by re-watering for 3 days. The hairy roots phenotype is shown in Figure 8D. Drought resistance of transgenic soybean plants was assayed by the capability to resume growth when returned to normal conditions after drought stress. Scale bar, $5 \mathrm{~cm}$. (E) Relative transcript of GmNFYA5 was detected in three transgenic soybean lines. Transcript of GmNFYA5 in EV plants was set at 1.0, and the expression levels were normalized to that of GmCYP2. (F-K) Survival rate, RWC, ion leakage, MDA, ABA concentration and SWP in transgenic soybean lines. Data represent mean SD for three biological replicates $(n=18)$. Significant differences $P<0.05$ are indicated by different letters above the columns. 

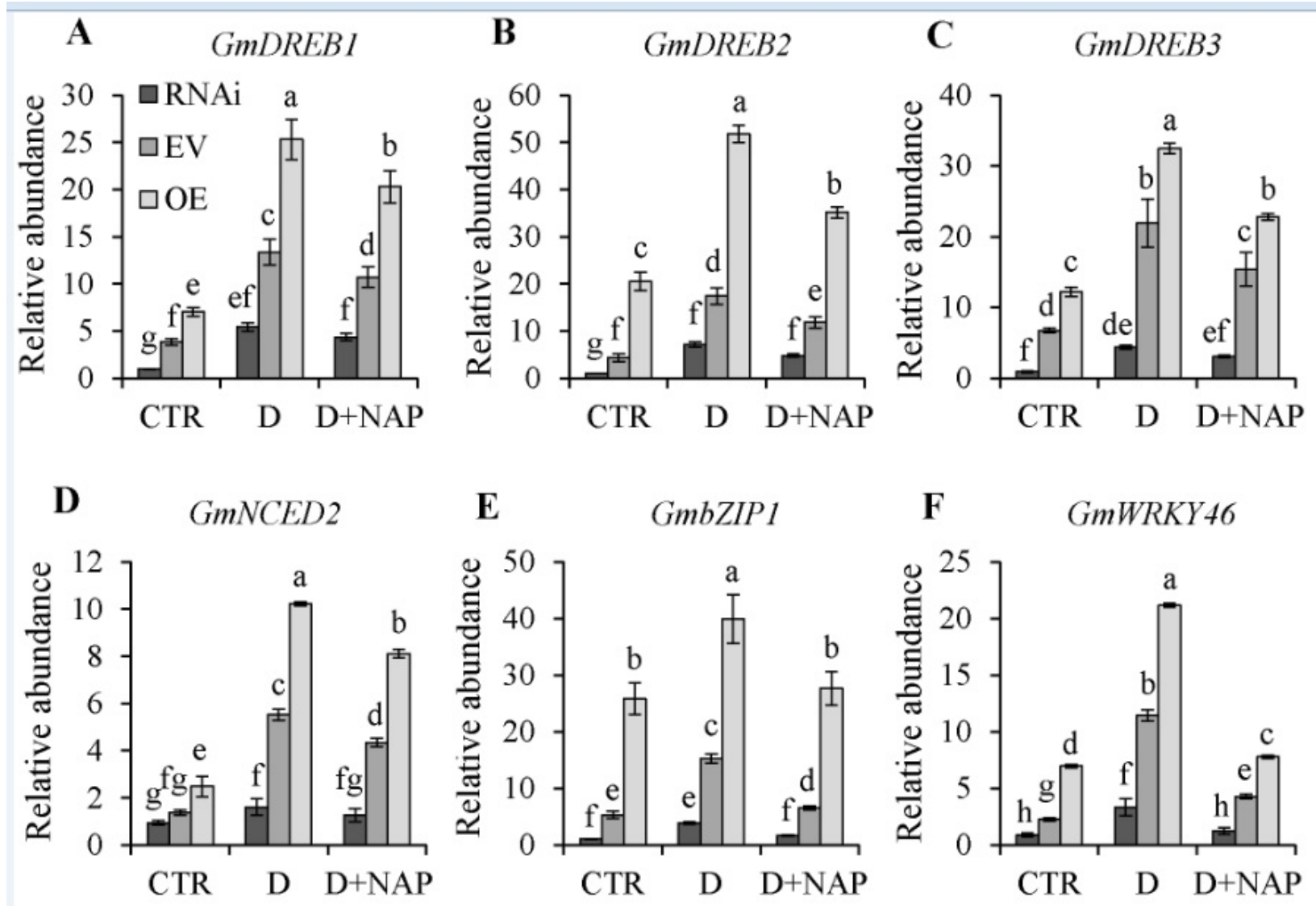

Figure 9

Relative transcript levels of GmDREB1, GmDREB2, GmDREB3, GmNCED2, GmWRKY46 and

GmbZIP1 in transgenic soybean and EV plants under three conditions. Relative transcript level is indicated by the vertical column and normalized to that of GmCYP2. Transcript level of stress-related genes in EV plants under normal condition was set at 1.0. Control, drought and drought + NAP are indicated by CTR, D and D + NAP, respectively. Data represent mean SD for three biological replicates. Significant differences $P<0.05$ are indicated by different letters above the columns. 
A

Effectors

Vector

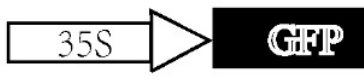

GmNFYA5

$35 \mathrm{~S}$

\section{GIP}

Reporters

GmDREB2P GmDREB2 promoter

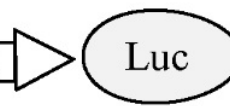

GmbZIP1P GmbZIPI promoter
B

Vector +

Vector +

GmDREB2P

GmNFYA5 + GmDREB2P

C

Vector + GmbZIP1P

GmNFYA5 + GmbZIP1P

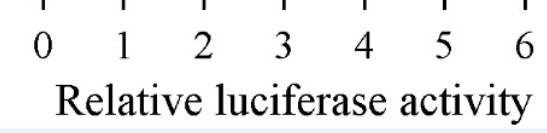

Figure 10

GmNFYA5 increased LUC reporter gene activity by binding the promoters of GmDREB2 and

GmbZIP1. (A) The structures of effector and reporters. (B) and (C) Ratio of LUC to REN indicates the activity of GmNFYA5 on the transcript of the GmDREB2 and GmbZIP1

promoters. Data represent mean SD for three biological replicates. Significant differences P $<0.05$ are indicated by different letters above the columns.

\section{Supplementary Files}

This is a list of supplementary files associated with this preprint. Click to download.

Figure S1.tif

Table S2.xlsx

Figure S3.tif

Figure S2.tif

Table S1.xlsx 